\title{
REVIEW
}

\section{Emission properties of nanolasers during the transition to lasing}

\author{
Weng W Chow ${ }^{1}$, Frank Jahnke ${ }^{2}$ and Christopher Gies ${ }^{2}$
}

This review addresses ongoing discussions involving nanolaser experiments, particularly those related to thresholdless lasing or few-emitter devices. A quantum-optical (quantum-mechanical active medium and radiation field) theory is used to examine the emission properties of nanolasers under different experimental configurations. The active medium is treated as inhomogeneously broadened semiconductor quantum dots embedded in a quantum well, where carriers are introduced via current injection. Comparisons are made between a conventional laser and a nanolaser with a spontaneous emission factor of unity, as well as a laser with only a few quantum dots providing the gain. It is found that the combined exploration of intensity, coherence time, photon autocorrelation function and carrier spectral hole burning can provide a unique and consistent picture of nanolasers in the new regimes of laser operation during the transition from thermal to coherent emission. Furthermore, by reducing the number of quantum dots in the optical cavity, a clear indication of non-classical photon statistics is observed before the single-quantum-dot limit is reached. Light: Science \& Applications (2014) 3, e201; doi:10.1038/lsa.2014.82; published online 29 August 2014

Keywords: nanolasers; optoelectronics; photon statistics; quantum light sources; quantum optics; semiconductor quantum dots; thresholdless lasing

\section{FROM CONVENTIONAL TO NANOLASERS}

Advances in nanofabrication have drastically reduced the size and increased the quality of optical cavities, enabling optical components with dimensions near the diffraction limit. ${ }^{1-3}$ Among the driving forces for substantial miniaturization of lasers are technological applications, such as optical interconnects for information processing and communications, where reducing the power consumption is a priority. ${ }^{4}$ From a research viewpoint, such efforts are motivated by a new quantum limit that is reachable with nanolasers consisting of a few emitters ${ }^{5-7}$ or even a single emitter ${ }^{8-10}$ and low intracavity photon numbers sustained by stimulated emission. ${ }^{11}$ Entering the regime of cavity quantum electrodynamics (CQED), nanolasers can provide non-classical light for applications in quantum information. ${ }^{12}$

Novel nano-optical structures, such as pillar vertical-cavity surfaceemitting lasers, microdisks, photonic lattices, nanowires and plasmonic resonators, ${ }^{13-16}$ enable the extension of optical-mode confinement from one-dimensional to three-dimensional (3D). The features of 3D mode confinement include spectrally widely separated cavity modes, allowing for the possibility of only one mode overlapping with the spontaneous emission spectrum. CQED phenomena include the enhancement ${ }^{17}$ or inhibition ${ }^{18}$ of spontaneous emission.

The spontaneous emission factor $\beta$ is a quantitative measure of optical resonator control over spontaneous emission. This factor is defined as the spontaneous emission rate into the laser mode divided by the total spontaneous emission rate. For small values of $\beta$, which are typical for conventional lasers, the onset of stimulated emission produces a distinct jump in output intensity. Recent advances in micro- and nanocavities with 3D optical mode confinement have led to $\beta$-factors approaching unity. In these cases, the intensity jump vanishes, which leads to the possibility of ultralow-threshold ${ }^{5-7}$ or even thresholdless lasers. ${ }^{1,19-21}$

The possibility of extremely high-quality ( $Q$-factor) nanocavities also allows for lasing with very few emitters in the active region. With a sufficiently small number of emitters, nanolasers producing non-classical light ${ }^{12}$ are being termed lasers. ${ }^{11,22}$ In the ultimate limit of a single emitter, lasing in the strong light-matter-coupling regime has also been recently achieved. ${ }^{10,23,24}$

Furthermore, a new research field has emerged from the use of plasmonic effects, ${ }^{25}$ which can lead to systems with drastically reduced optical mode volume and increased light-matter coupling efficiency compared to purely photonic systems. These prospects have stimulated work on plasmonic lasers, ${ }^{16,26}$ culminating in the recent demonstration of a self-assembled quantum-dot (QD) transition coupled to a plasmonic resonance. ${ }^{27}$ With an appropriate choice of parameter values, e.g., for the confinement factor and the intracavity optical loss, the theoretical approach described in this review applies equally well to these new and exciting systems, as well as to conventional photonic lasers.

\section{LASING CRITERIA}

Historically, lasing has been understood in many ways, ranging from amplification driven by stimulated emission ${ }^{28}$ to a feedback process leading to oscillations. ${ }^{29}$ These descriptions lead to the phenomenon of a lasing threshold, where an abrupt transition occurs in the laser

${ }^{1}$ Sandia National Laboratories, Albuquerque, NM 87185-1086, USA and ${ }^{2}$ Institute for Theoretical Physics, University of Bremen, 28334 Bremen, Germany

Correspondence: Dr WW Chow, Sandia National Laboratories, Albuquerque, NM 87185-1086, USA

E-mail: wwchow@sandia.gov

Received 27 March 2014; revised 17 May 2014; accepted 20 May 2014 
intensity and noise. This abruptness has been studied in terms of a phase transition. ${ }^{3,30}$ Both experiment and theory indicate that the traditional concepts of laser action must be re-examined for nanolasers. For example, there is debate on the criteria for lasing and lasing thresholds in the absence of an intensity jump. ${ }^{2,3,29,31}$ Additionally, few-emitter nanolasers exhibit sub-Poisson photon statistics, in contrast to the Poisson photon statistics dictated by the randomness of spontaneous emission after the onset of lasing. ${ }^{12}$

It is widely agreed that a careful examination of photon statistics is necessary. A discussion in terms of well-established laser rate equations ${ }^{1,32-34}$ is unable to provide the photon statistics or to capture the semiconductor effects that strongly determine device behavior. A fully quantum-mechanical laser model is necessary to investigate in addition to the changes in the intracavity photon number also the first- and second-order photon correlation functions. The latter provides a description of the quantum optical properties of light, distinguishing between thermal, coherent and non-classical emission.

\section{QD NANOLASERS}

To develop a microscopic theory for predicting photon statistics under new regimes of nanolaser operation, we consider an active medium consisting of semiconductor QDs. Self-assembled Stranski-Krastanov semiconductor QDs are increasingly used in semiconductor lasers and are strong candidates for replacing quantum wells (QWs) in future generations of ultrahigh-efficiency optoelectronic devices. ${ }^{35,36}$ The 3D carrier confinement in a QD causes strong localization of electronhole pairs, resulting in high emission efficiency. Additionally, Stranski-Krastanov QDs are embedded in semiconductor materials. Interactions between the carriers in the localized QD states and those in the continuum states of the wetting layer and barrier materials result in fast scattering processes and enable efficient carrier generation, especially via electrical pumping, which is highly desirable for optoelectronic applications.

The general findings from the model regarding nanolaser operation in the limit of $\beta=1$ should apply to both QW and bulk active regions. Our investigations involve a comparison between a laser with a welldefined threshold and the ideal nanolaser with $\beta=1$. The results are analyzed in terms of the intracavity photon number, coherence time and second-order photon correlation versus pump rate for different cavity photon decay rates. We will also look for evidence of carrier population clamping and hole burning in inhomogeneously broadened QD distributions.

One reason for our choice of a QD active region is that it allows for an investigation of the effect of reducing the number of emitters to produce non-classical light. A single QD in a 3D nanocavity represents the miniaturization limit and the crossover domain to non-classical light sources, which are of high relevance for quantum information applications. We would like to investigate nanolaser behavior in the few-emitter limit, where several experiments have been performed, ${ }^{5-7}$ and to determine whether novel emission properties arise, including non-classical light generation, without reaching the single-QD limit.

\section{NANOLASER MODEL INCORPORATING SEMICONDUCTOR QUANTUM OPTICS}

In this section, we describe the development of a model for a currentdriven, semiconductor QD, nanocavity laser for computing the intracavity photon number, coherence time and second-order intensity correlation (see Figure 1 for an overview). The model is based on a previously developed semiconductor CQED theory ${ }^{37}$ and improves on the rate-equation description typically used to illustrate the effects

a Cavity-quantum-electrodynamics

a Cavity-quantum-electrodynamics nanolaser model Hanbury-Brown-Twiss experiment
b

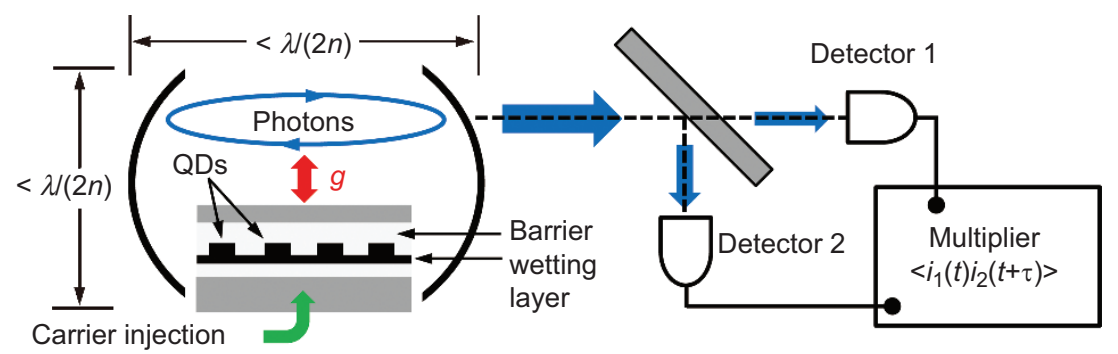

Nanocavity

d

Nanolaser emission
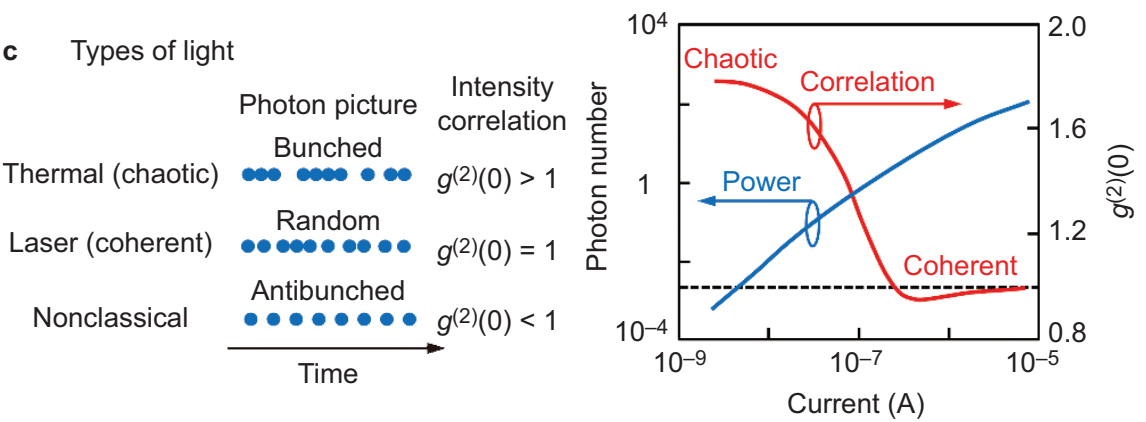

Figure 1 (a) Sketch of a generic nanolaser used in developing the quantum-electrodynamics model. The basic features include an active medium inside an optical cavity with dimensions of less than $(\lambda / 2 n)^{3}$, where $\lambda$ is the lasing wavelength and $n$ is the background refractive index. Central to the physics is the treatment of lightmatter correlations (see the red double arrow), characterized by the coupling coefficient $g$ (Equation (2)). (b) Hanbury-Brown-Twiss setup for determining the intensity correlation, which is proportional to the product of the photocurrents, i.e., $g^{(2)}(0) \propto\left\langle i_{1}(t) i_{2}(t)\right\rangle$. (c) Different types of light classified by a Hanbury-Brown-Twiss measurement. (d) A possible combination of observables providing precise information on lasing in an experiment with $\beta=1$, where complete channeling of spontaneous emission into the lasing mode is realized. QD, quantum dot. 
of $\beta$ in atomic-molecular-optical systems. ${ }^{1,32}$ This aim is accomplished with a consistent derivation of photon correlations along with the equations of motion for carrier and photon populations. We also include a description of QD excitation via the surrounding QWs in terms of carrier injection, capture and escape, as well as inhomogeneous broadening due to QD size and composition fluctuations.

We consider an active medium consisting of $\operatorname{In}_{0.3} \mathrm{Ga}_{0.7} \mathrm{As}$ QDs and a wetting layer embedded in a GaAs QW. By assuming a cylindrical QD shape 2-nm tall and 18-nm in diameter, the electronic structure calculation $^{36,38}$ predicts only one electron bound state and one hole bound state because of the shallow quantum confinement. This simple electronic structure is well suited for illustrating the nanolaser behaviors of interest.

To derive the equations of motion, we use the Jaynes-Cummings Hamiltonian $^{39}$ for the light-matter interaction, with commutation relations for the photon operators and anti-commutation relations for the electron and hole operators. In the dipole and rotating-wave approximations, the Hamiltonian describing the light-matter interaction has the form:

$H=\hbar v\left(b^{\dagger} b+\frac{1}{2}\right)+\sum_{n}\left[\varepsilon_{\mathrm{e}, n}^{\mathrm{QD}} c_{n}^{\dagger} c_{n}+\varepsilon_{\mathrm{h}, n}^{\mathrm{QD}} v_{n}^{\dagger} v_{n}-i \hbar\left(g b c_{n}^{\dagger} v_{n}-g^{*} b^{\dagger} v_{n}^{\dagger} c_{n}\right)\right](1)$

where $\hbar v$ is the photon energy, $b^{\dagger}$ and $b$ are the photon creation and annihilation operators, $\varepsilon_{\mathrm{e}, n}^{\mathrm{QD}}$ and $\varepsilon_{\mathrm{h}, n}^{\mathrm{QD}}$ are the single-particle electron and hole energies of the $n^{\text {th }} \mathrm{QD}, c_{n}^{\dagger}$ and $c_{n}$ are the creation and annihilation operators for conduction-band electrons, $v_{n}^{\dagger}$ and $v_{n}$ are the corresponding operators for valence-band electrons, and the summation is taken over the inhomogeneous QD distribution. The light-matter coupling coefficient is:

$$
g=\wp \sqrt{\frac{v}{\hbar \epsilon_{b} V}} W\left(\boldsymbol{R}_{\mathrm{QD}}\right) \sum_{n} C\left(\boldsymbol{R}_{n}\right) V\left(\boldsymbol{R}_{n}\right)
$$

where $\wp$ is the bulk material dipole matrix element, $V$ is the optical mode volume, $W$ is the amplitude of the passive optical mode eigenfunction at $\boldsymbol{R}_{\mathrm{QD}}$, the location of the QDs within the optical cavity, and the summation involves the overlap of electron and hole envelope functions over all unit cells within the active region. The light-matter coupling coefficient $g$ can also be determined experimentally.

Working in the Heisenberg picture, we derive equations of motion for the polarization $p_{n}=\left\langle b c_{n}^{\dagger} v_{n}\right\rangle$, photon population $n_{\mathrm{p}}=\left\langle b^{\dagger} b\right\rangle$ and carrier populations $n_{\mathrm{e}, n}^{\mathrm{QD}}=\left\langle c_{n}^{\dagger} c_{n}\right\rangle$ and $n_{\mathrm{h}, n}^{\mathrm{QD}}=1-\left\langle v_{n}^{\dagger} v_{n}\right\rangle$, respectively:

$\frac{\mathrm{d} p_{n}}{\mathrm{~d} t}=-\left[i\left(v-\omega_{n}\right)+\left(\gamma+\gamma_{\mathrm{c}}\right)\right] p_{n}+g^{*}\left[n_{\mathrm{e}, n}^{\mathrm{QD}} n_{\mathrm{h}, n}^{\mathrm{QD}}+\left(n_{\mathrm{e}, n}^{\mathrm{QD}}+n_{\mathrm{h}, n}^{\mathrm{QD}}-1\right) n_{\mathrm{p}}\right](3)$

$$
\frac{\mathrm{d} n_{\mathrm{p}}}{\mathrm{d} t}=2 \sum_{n} n_{\mathrm{inh}}\left(\omega_{n}\right) \operatorname{Re}\left(g p_{n}\right)-2 \gamma_{\mathrm{c}} n_{\mathrm{p}}
$$

$$
\begin{aligned}
\frac{\mathrm{d} n_{\sigma, n}^{\mathrm{QD}}}{\mathrm{d} t}= & -2 \operatorname{Re}\left(g p_{n}\right)-\gamma_{\mathrm{nl}} n_{\mathrm{e}, n}^{\mathrm{QD}} n_{\mathrm{h}, n}^{\mathrm{QD}}-\gamma_{\mathrm{nr}} n_{\sigma, n}^{\mathrm{QD}} \\
& -\gamma_{\mathrm{c}-\mathrm{c}}\left[n_{\sigma, n}^{\mathrm{QD}}-f\left(\varepsilon_{\sigma, n}^{\mathrm{QD}}, \mu_{\sigma}, T\right)\right]-\gamma_{\mathrm{c}-\mathrm{p}}\left[n_{\sigma, n}^{\mathrm{QD}}-f\left(\varepsilon_{\sigma, n}^{\mathrm{QD}}, \mu_{\sigma}^{1}, T_{1}\right)\right]
\end{aligned}
$$

where $2 \gamma_{\mathrm{c}}$ is the photon decay rate in the cavity, $\gamma_{\mathrm{nl}}$ is the spontaneous emission rate into non-lasing modes, $\gamma_{\mathrm{nr}}$ accounts for non-radiative carrier loss and $\sigma=e(h)$ indicates electron (hole). Inhomogeneous broadening due to QD dimension or composite non-uniformity is modeled by including a QD population distribution in Equation (4):

$$
n_{\mathrm{inh}}\left(\omega_{n}\right)=\frac{N_{\mathrm{QD}}}{\sqrt{2 \pi} \Delta_{\mathrm{inh}}} \exp \left[-\left(\frac{\hbar\left(\omega_{n}-\omega_{0}\right)}{\sqrt{2} \Delta_{\mathrm{inh}}}\right)^{2}\right]
$$

where $N_{\mathrm{QD}}$ is the total number of QDs in the active region, $\omega_{0}$ is the central transition frequency of the inhomogeneous distribution and $\Delta_{\text {inh }}$ is the inhomogeneous width. We group the QDs into bins of width $\hbar \gamma$, each with an average transition frequency energy $\omega_{n}$ and QD number $n_{\text {inh }}\left(\omega_{n}\right)$. The closed set of Equations (3)-(5) is obtained from the operator equations by using a correlation function expansion, ${ }^{36,37,40}$ keeping only correlations at the doublet level. The limitations that arise from neglecting higher-order light-matter correlations will be discussed later, when we look at few-QD emitters.

Pumping of the QD states takes place via the QW carrier population $n_{\sigma, k}^{\mathrm{QW}}$ :

$$
\begin{aligned}
& \frac{\mathrm{d} n_{\sigma, k}^{\mathrm{QW}}}{\mathrm{d} t}=\frac{I}{e N_{\sigma}^{\mathrm{p}}} f\left(\varepsilon_{\sigma, k}^{\mathrm{QW}}, \mu_{\sigma}^{\mathrm{p}}, T_{\mathrm{p}}\right)\left(1-n_{\sigma, k}^{\mathrm{QW}}\right)-\gamma_{\mathrm{nr}} n_{\sigma, k}^{\mathrm{QW}} \\
& -\gamma_{\mathrm{c}-\mathrm{c}}\left[n_{\sigma, k}^{\mathrm{QW}}-f\left(\varepsilon_{\sigma, k}^{\mathrm{QW}}, \mu_{\sigma}, T\right)\right]-\gamma_{\mathrm{c}-\mathrm{p}}\left[n_{\sigma, k}^{\mathrm{QW}}-f\left(\varepsilon_{\sigma, k}^{\mathrm{QW}}, \mu_{\sigma}^{\mathrm{l}}, T_{1}\right)\right]
\end{aligned}
$$

where $k$ is the two-dimensional carrier momentum, $I$ is the current, $e$ is the electron charge and

$$
N_{\sigma}^{\mathrm{p}}=\sum_{k} f\left(\varepsilon_{\sigma, k}^{\mathrm{QW}}, \mu_{\sigma}^{\mathrm{p}}, T_{\mathrm{p}}\right)
$$

is the steady-state QW carrier population created by the injection current when all radiative processes are switched off. The injected carrier distribution $f\left(\varepsilon_{\sigma, k}^{\mathrm{QW}}, \mu_{\sigma}^{\mathrm{p}}, T_{\mathrm{p}}\right)$ is assumed to be a Fermi-Dirac function with chemical potential $\mu_{\sigma}^{\mathrm{p}}$ and temperature $T_{\mathrm{p}}$.

Scattering effects lead to polarization dephasing, as well as carrier capture and escape. The model accounts for these effects using a phenomenological approach ${ }^{41}$ that has been shown to reproduce the results of quantum kinetic treatments ${ }^{42}$ when carrier-carrier and carrier-phonon scattering rates are distinctly different. This is the case for electrically driven devices, where the carrier-phonon scattering rate is roughly an order of magnitude lower than that of carrier-carrier scattering. In this situation, carrier-carrier scattering first tries to drive a non-equilibrium carrier distribution to a Fermi-Dirac distribution given by some chemical potential and plasma temperature, $\mu_{\sigma}$ and $T$, respectively. Then, the carrier-phonon scattering further relaxes the carriers to another Fermi-Dirac function given by chemical potential $\mu_{\sigma}^{1}$ and lattice temperature $T_{1}$.

The above description is included in the above equations via the terms containing $\gamma$ (dephasing rate), $\gamma_{\mathrm{c}-\mathrm{c}}$ (carrier-carrier scattering rate) and $\gamma_{c-p}$ (carrier-phonon scattering rate). The asymptotic Fermi-Dirac distributions approached through carrier-carrier scattering are $f\left(\varepsilon_{\sigma, n}^{\mathrm{QD}}, \mu_{\sigma}, T\right)$ and $f\left(\varepsilon_{\sigma, k}^{\mathrm{QW}}, \mu_{\sigma}, T\right)$. Their chemical potential $\mu_{\sigma}$ and plasma temperature $T$ are computed by the conservation of carrier population and energy. For asymptotic distributions associated with carrier-phonon scattering, $f\left(\varepsilon_{\sigma, n}^{\mathrm{QD}}, \mu_{\sigma}^{1}, T_{1}\right)$ and $f\left(\varepsilon_{\sigma, k}^{\mathrm{QW}}, \mu_{\sigma}^{1}, T_{1}\right)$, the chemical potential $\mu_{\sigma}^{1}$ is obtained from the conservation of carrier population, where the lattice temperature $T_{1}$ is an input quantity.

As mentioned in the introduction, to address issues regarding lasing thresholds, one must examine the photon statistics. Here, we look at the coherence time and the second-order correlation function. The coherence time of the radiation in the stationary situation is given by: 


$$
\tau_{\mathrm{c}}=2 \int_{-\infty}^{\infty} \mathrm{d} \tau\left|g^{(1)}(\tau)\right|^{2}
$$

where

$$
g^{(1)}(\tau)=\frac{\left\langle b^{\dagger} b(\tau)\right\rangle_{\mathrm{ss}}}{n_{\mathrm{p}}^{\mathrm{ss}}}
$$

with ss indicating that the expectation values are computed using the steady-state values for $n_{\mathrm{p}}, p_{n}, n_{\mathrm{e}, n}^{\mathrm{QD}}$ and $n_{\mathrm{h}, n}^{\mathrm{QD}}$. To derive the equation of motion for $\left\langle b^{\dagger} b(\tau)\right\rangle$, we again work in the Heisenberg picture and use the Hamiltonian described by Equation (1). This gives:

$$
\begin{gathered}
\frac{\mathrm{d} G^{(1)}}{\mathrm{d} \tau}=-\gamma_{\mathrm{c}} G^{(1)}+2 \sum_{n} n_{\mathrm{inh}}\left(\omega_{n}\right) \operatorname{Re}\left(g^{*} P_{n}\right) \\
\frac{\mathrm{d} P_{n}}{\mathrm{~d} \tau}=\left[i\left(v-\omega_{n}\right)-\left(\gamma+\gamma_{\mathrm{c}}\right)\right] P_{n}+g^{*}\left(n_{\mathrm{e}, n}^{\mathrm{QD}}+n_{\mathrm{h}, n}^{\mathrm{QD}}-1\right)_{\mathrm{ss}} G^{(1)}
\end{gathered}
$$

where we factored out the rapid oscillations in $\tau$ by defining $G^{(1)}(\tau)=\left\langle b^{\dagger} b(\tau)\right\rangle_{\mathrm{ss}} e^{i v \tau}$ and $P_{n}(\tau)=\left\langle b^{\dagger} v_{n}^{\dagger}(\tau) c_{n}(\tau)\right\rangle_{\mathrm{ss}} e^{i v \tau}$, as discussed in Ref. 43.

For the equal-time second-order correlation,

$$
g^{(2)}(0)=\frac{\left\langle b^{\dagger} b^{\dagger} b b\right\rangle}{n_{\mathrm{p}}^{2}}
$$

where we introduce the following expectation values:

$$
\begin{gathered}
\delta\left\langle b^{\dagger} b^{\dagger} b b\right\rangle=\left\langle b^{\dagger} b^{\dagger} b b\right\rangle-2 n_{\mathrm{p}}^{2} \\
\delta\left\langle b^{\dagger} b^{\dagger} b v_{n}^{\dagger} c_{n}\right\rangle=\left\langle b^{\dagger} b^{\dagger} b v_{n}^{\dagger} c_{n}\right\rangle-n_{\mathrm{p}}\left(p_{n}\right)^{*} \\
\delta\left\langle b^{\dagger} b c_{n}^{\dagger} c_{n}\right\rangle=\left\langle b^{\dagger} b c_{n}^{\dagger} c_{n}\right\rangle-n_{\mathrm{p}} n_{\mathrm{e}, n}^{\mathrm{QD}}
\end{gathered}
$$

and

$$
\delta\left\langle b^{\dagger} b v_{n}^{\dagger} v_{n}\right\rangle=\left\langle b^{\dagger} b v_{n}^{\dagger} v_{n}\right\rangle-n_{\mathrm{p}}\left(1-n_{\mathrm{h}, n}^{\mathrm{QD}}\right)
$$

Continuing with the derivation to the quadruplet level, we obtain the necessary equations of motion:

$$
\begin{aligned}
& \frac{\mathrm{d} \delta\left\langle b^{\dagger} b^{\dagger} b b\right\rangle}{\mathrm{d} t}= \\
& -4 \gamma_{\mathrm{c}} \delta\left\langle b^{\dagger} b^{\dagger} b b\right\rangle+4 \sum_{n} n_{\mathrm{inh}}\left(\omega_{n}\right) \operatorname{Re}\left(g^{*} \delta\left\langle b^{\dagger} b^{\dagger} b v_{n}^{\dagger} c_{n}\right\rangle\right) \\
& \frac{\mathrm{d} \delta\left\langle b^{\dagger} b^{\dagger} b v_{n}^{\dagger} c_{n}\right\rangle}{\mathrm{d} t}=-\left(\gamma+3 \gamma_{c}\right) \delta\left\langle b^{\dagger} b^{\dagger} b v^{\dagger}{ }_{n} c_{n}\right\rangle \\
& +g^{*}\left(n_{\mathrm{e}, n}^{\mathrm{QD}}+n_{\mathrm{h}, n}^{\mathrm{QD}}-1\right) \delta\left\langle b^{\dagger} b^{\dagger} b b\right\rangle \\
& +2 g^{*}\left[\left(n_{\mathrm{h}, n}^{\mathrm{QD}}+n_{\mathrm{p}}\right) \delta\left\langle b^{\dagger} b c_{n}^{\dagger} c_{n}\right\rangle\right. \\
& \left.-\left(n_{\mathrm{e}, n}^{\mathrm{QD}}+n_{\mathrm{p}}\right) \delta\left\langle b^{\dagger} b v_{n}^{\dagger} v_{n}\right\rangle\right]-2 g^{*} p_{n}^{2} \\
& \frac{\mathrm{d} \delta\left\langle b^{\dagger} b c_{n}^{\dagger} c_{n}\right\rangle}{\mathrm{d} t}=-2 \gamma_{\mathrm{c}} \delta\left\langle b^{\dagger} b c_{n}^{\dagger} c_{n}\right\rangle- \\
& 2 \operatorname{Re}\left[\left(n_{\mathrm{e}, n}^{\mathrm{QD}}+n_{\mathrm{p}}\right) g p_{n}+g^{*} \delta\left\langle b^{\dagger} b^{\dagger} b v_{n}^{\dagger} c_{n}\right\rangle\right]
\end{aligned}
$$

$$
\begin{aligned}
& \frac{\mathrm{d} \delta\left\langle b^{\dagger} b v_{n}^{\dagger} v_{n}\right\rangle}{\mathrm{d} t}=-2 \gamma_{\mathrm{c}} \delta\left\langle b^{\dagger} b v_{n}^{\dagger} v_{n}\right\rangle+ \\
& 2 \operatorname{Re}\left[\left(n_{\mathrm{h}, n}^{\mathrm{QD}}+n_{\mathrm{p}}\right) g p_{n}+g^{*} \delta\left\langle b^{\dagger} b^{\dagger} b v_{n}^{\dagger} c_{n}\right\rangle\right]
\end{aligned}
$$

The calculation inputs include the light-matter coupling strength, carrier and photon loss rates, and capture and relaxation rates, which are given in terms of effective carrier-carrier and carrier-phonon relaxation coefficients. Assuming a mode volume of $\left(\lambda / 2 n_{\mathrm{B}}\right)^{3}$, where $\lambda$ is the emitter wavelength and $n_{\mathrm{B}}$ is the background refractive index, and a dipole matrix element of $e \times 0.5 \mathrm{~nm}$, the light-matter coupling coefficient is $g=2.68 \times 10^{11} \mathrm{~s}^{-1}$. The remaining relaxation and loss coefficients are $\gamma=10^{13} \mathrm{~s}^{-1}, \gamma_{\mathrm{nr}}=10^{9} \mathrm{~s}^{-1}, \gamma_{\mathrm{c}-\mathrm{c}}=10^{13} \mathrm{~s}^{-1}$ and $\gamma_{\mathrm{c}-\mathrm{p}}=10^{12} \mathrm{~s}^{-1}$. From the electronic structure calculation, the QD electron and hole states are $40 \mathrm{meV}$ and $30 \mathrm{meV}$ below their respective QW band edges. In the following figures, $\gamma_{\mathrm{nl}}=0$ and $\gamma_{\mathrm{nl}}=1.4 \times 10^{12} \mathrm{~s}^{-1}$ are used to give $\beta=1$ and $\beta=0.01$, respectively. $\beta$ is determined via

$$
\beta=\frac{2 \sum_{n} \operatorname{Re}\left(g p_{n}^{\prime}\right)_{\mathrm{ss}}}{\sum_{n}\left[2 \operatorname{Re}\left(g p_{n}^{\prime}\right)_{\mathrm{ss}}+\gamma_{\mathrm{nl}} n_{\mathrm{e}, n}^{\mathrm{QD}} n_{\mathrm{h}, n}^{\mathrm{QD}}\right]}
$$

where

$$
\frac{\mathrm{d} p_{n}^{\prime}}{\mathrm{d} t}=g^{*} n_{\mathrm{e}, n}^{\mathrm{QD}} n_{\mathrm{h}, n}^{\mathrm{QD}}
$$

is solved together with Equations (3)-(6) until a steady state is reached.

We note that in rate-equation models, $\beta$ is typically a free parameter. In the microscopic theory, the rates of emission into the laser mode and into non-lasing modes are explicitly determined according to the system parameters, and the $\beta$-factor follows accordingly. For a homogeneously broadened QD sample $\left(\Delta_{\text {inh }}=0\right)$, the value of $\beta$, evaluated in this manner, is excitation-independent. Lastly, using $n_{\mathrm{p}}, p_{n}$, $n_{\mathrm{e}, n}^{\mathrm{QD}}$ and $n_{\mathrm{h}, n}^{\mathrm{QD}}$, we evaluate Equations (9)-(21) for the coherence time and the equal-time second-order correlation.

We end this section by illustrating some differences between previous models ${ }^{1,32,37}$ and the present one, which incorporates the details of a self-assembled Stranski-Krastanov semiconductor QD active region. Figure 2a presents a comparison of the intracavity photon number versus injection current. The black curve shows the result from an earlier QD-CQED model, ${ }^{37}$ which, for the parameters used, basically reproduces the familiar rate equation result for the mean photon number versus pump rate of the QD states (which, in this case, is assumed to be directly proportional to the injection current). The blue curve represents the result of including the QW states, where electron and hole injection occur, and the red curve includes an additional QD inhomogeneous broadening, $\Delta_{\text {inh }}=20 \mathrm{meV}$. All curves are computed for a 50-QD sample with a cavity loss rate $\gamma_{c}=10^{10} \mathrm{~s}^{-1}$. One difference lies in the threshold current between the black curve and the other curves, originating from the carrier injection being moved from directly into the QD levels to the QW states. A further difference occurs in terms of the output saturation at a high pump rate arising from the reduction in the number of QDs effectively interacting with the cavity photons due to inhomogeneous broadening. This effect is shown more clearly in the inset, where the curves are superimposed on each other by referencing the current to the respective threshold currents. The photon number rollover distorts the familiar ' $S$ ' shape, which complicates the common practice of extracting $\beta$ from a log$\log$ plot of output versus input, using rate equations and assuming $\beta$ to be independent of the excitation. 

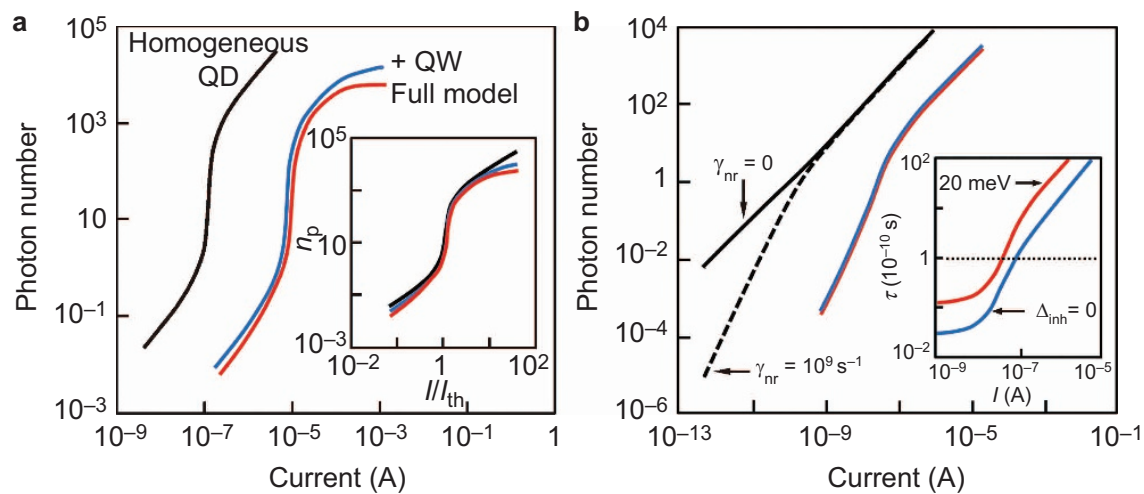

Figure 2 Photon number versus injection current for 50 QDs in a cavity with a photon loss rate $\gamma_{c}=10^{10} \mathrm{~s}^{-1}$ and spontaneous emission factor $\beta=0.01$ (a) and 1 (b). The black curves are obtained using a QD-CQED model without QWs or inhomogeneous broadening and with non-radiative carrier loss rates $\gamma_{\mathrm{nr}}=0$ and $10^{9} \mathrm{~s}^{-1}$ ( $\mathrm{solid}$ and dashed curves, respectively). The blue curves include a QW in which carriers are injected. The red curves further add QD inhomogeneous broadening, $\Delta_{\mathrm{inh}}=20 \mathrm{meV}$. The inset in a shows a comparison of the saturation behavior by normalizing the current to lasing threshold values. The inset in $\mathbf{b}$ reveals a significant difference in coherence time in the presence of inhomogeneous broadening. CQED, cavity quantum electrodynamics; QD, quantum dot; QW, quantum well.

Figure $2 \mathrm{~b}$ illustrates the same comparison for $\beta=1$. The plot shows that the introduction of non-radiative carrier loss, as well as pumping via the QW states, causes a deviation from the linear input-output behavior predicted from the rate equations for the 'thresholdless' $\beta=1$ limit. QD inhomogeneous broadening of $\Delta_{\text {inh }}=20 \mathrm{meV}$ does not appreciably alter the photon number or $g^{(2)}(0)$ dependence (not shown) on injection current. However, the effect of broadening on the coherence time is significant, as illustrated in the inset in Figure 2b.

\section{EMISSION AND COHERENCE OF NANOLASERS}

We now apply the model described in the previous section to analyze the emission of a laser with a well-defined threshold and that of the ideal nanolaser with $\beta=1$. The comparison is provided in terms of the intracavity photon number, coherence time and second-order photon correlation versus pump rate. Different cavity losses are chosen to illustrate the device operation as a laser, a cavity-enhanced LED and an LED.

Figure 3a shows the input-output curves for $\beta=0.01$. With sufficiently low cavity losses, lasing is possible, as indicated by the ' $\mathrm{S}$ ' shape input-output photon number dependence (black and blue curves). With the greater cavity losses, the photon number jump vanishes and the devices remain below the lasing threshold indefinitely (red and green curves). As shown in Figure 3b, the equal-time second-order photon correlation changes from essentially 2 to 1 in the transition to lasing for the curves with the two lowest cavity losses. Figure $3 \mathrm{c}$ presents the injection current dependence of the coherence time. The product of coherence time and cavity loss is used to enable the curves to be superimposed for better comparison. For $\gamma_{c}=5 \times 10^{11} \mathrm{~s}^{-1}$, the cavity effects are weak, as evidenced by the vanishing injection current dependences of the second-order correlation and coherence time. Moreover, a further increase in $\gamma_{c}$ produces very little change from the $\gamma_{\mathrm{c}}=5 \times 10^{11} \mathrm{~s}^{-1}$ results (not shown). Proceeding from an LED towards lasing diodes, we note that below the lasing threshold, the cavity effects lead to a decrease in coherence time below that given by the passive cavity. This decrease translates to an increase in emission bandwidth or emission rate, a signature of Purcell enhancement (see the black curve in Figure $2 \mathrm{c}) .{ }^{17}$

Figure 4 illustrates the differences that arise when the spontaneous emission factor is $\beta=1$. Here, the ' $\mathrm{S}$ ' shape in the input-output curve disappears, as is well known. There have been considerable discussions on emission properties, e.g., involving thresholdless lasers or perhaps infinite threshold devices. Figure $4 \mathrm{~b}$ indicates that while the transition is somewhat smoother, there still exist regions with distinct emission properties. In the case of the second order correlation, a low-current regime is observed, where $g^{(2)}(0)$ is closer to the thermal radiation value of 2 than the laser value of 1 , as well as the lasing regime (if the lasing threshold is reachable), where $g^{(2)}(0)=1$. The red curve shows the case that may be the closest to an infinite threshold laser in that $g^{(2)}(0)$ approaches, but never reaches, unity. Here, the cavity losses are sufficiently high such that stimulated emission never dominates spontaneous emission. The coherence length behavior in Figure $4 \mathrm{c}$ basically mirrors that of Figure $3 c$, with significant increases
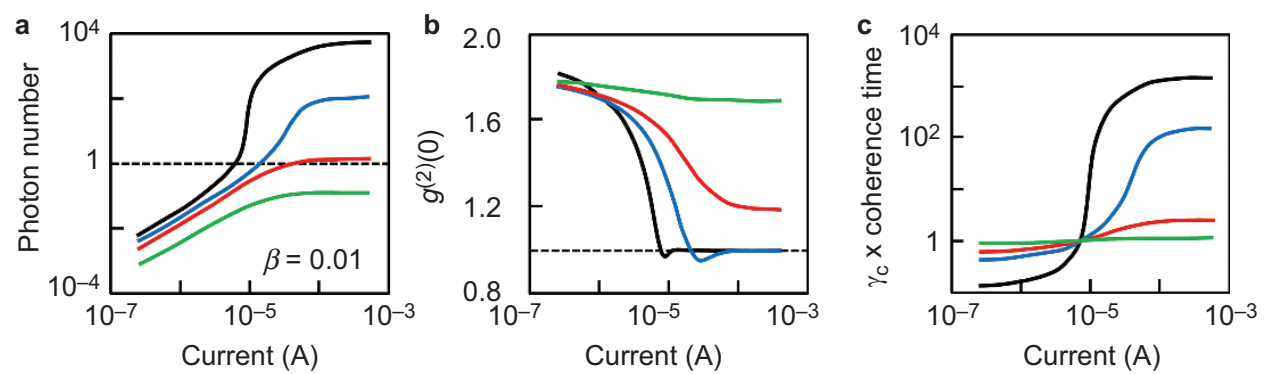

Figure 3 Injection current dependences of (a) photon number, (b) equal-time second-order photon correlation and (c) coherence time for an emitter consisting of 50 QDs in a cavity with a spontaneous emission factor $\beta=0.01$. The different curves correspond to photon loss rates $\gamma_{\mathrm{c}}=10^{10} \mathrm{~s}^{-1}, 5 \times 10^{10} \mathrm{~s}^{-1}, 10^{11} \mathrm{~s}^{-1}$ and $5 \times 10^{11} \mathrm{~s}^{-1}$. QD, quantum dot. 

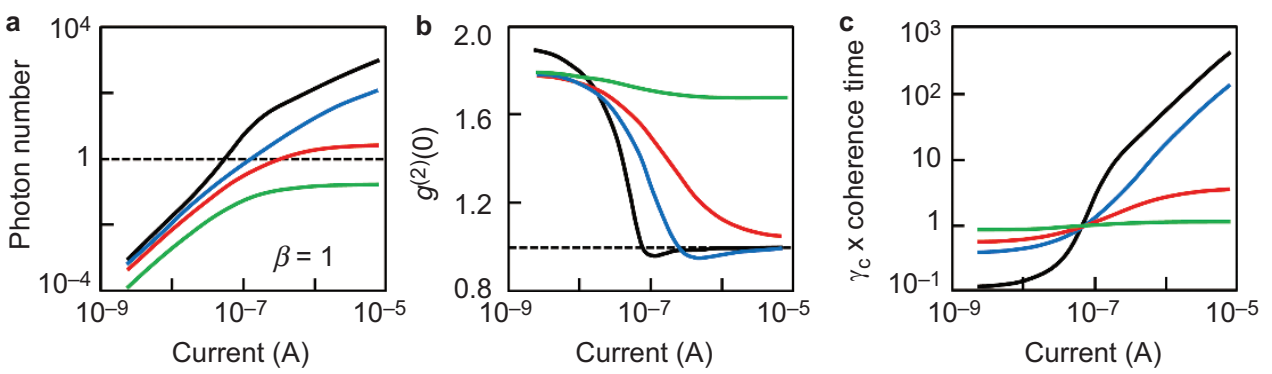

Figure 4 Injection current dependences of (a) photon number, (b) equal-time second-order photon correlation and (c) coherence time for an emitter consisting of 50 QDs in a cavity with a spontaneous emission factor $\beta=1$. The different curves correspond to photon loss rates $\gamma_{\mathrm{c}}=10^{10} \mathrm{~s}^{-1}, 5 \times 10^{10} \mathrm{~s}^{-1}, 10^{11} \mathrm{~s}^{-1}$ and $5 \times 10^{11} \mathrm{~s}^{-1}$. $\mathrm{QD}$, quantum dot.

in the two lasing cases, while smaller or no changes are observed for the LED and cavity-enhanced LED configurations. Hence, while Figures $3 a$ and 4 a show a significant change in the input-output behavior, with the loss of indication of a lasing threshold, the second-order photon correlation and coherence times maintain transition regions, indicating a crossover from thermal to coherent operation.

Additional insight may be gained by combining the photon number and $g^{(2)}(0)$ plots to give $g^{(2)}(0)$ versus photon number. Figure 5a shows that the transition from thermal to coherent operation depends only on the photon number and is independent of $\beta$. This result arises because the photon statistics are determined by the relative contributions from spontaneous and stimulated emission. When the former is dominant, thermal emission occurs, and when the latter prevails, coherent radiation is realized. For stimulated emission to dominate, the photon number per mode must be greater than one. Different $\beta$ values result in differences in the currents that are necessary to reach this condition.
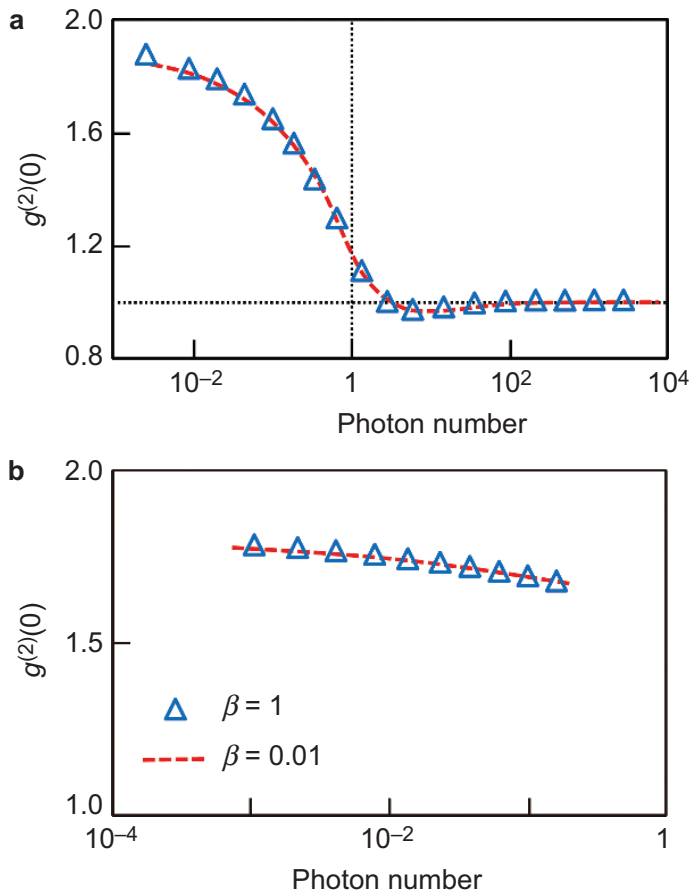

Figure 5 Equal-time second-order photon correlation versus photon number for $\beta=0.01$ and 1.0 (dashed curve and triangles, respectively). The photon loss rates are $\gamma_{\mathrm{c}}=10^{10} \mathrm{~s}^{-1}(\mathbf{a})$ and $5 \times 10^{11} \mathrm{~s}^{-1}(\mathbf{b})$.
The above argument is reinforced by Figure $5 b$, where, again, the $\beta=0.01$ and $\beta=1$ curves overlap each other. Here, the active material with a given QD number cannot produce enough gain to compensate the cavity losses, even if all emitters are fully inverted at high injection currents. Hence, spontaneous emission prevails, the mean photon number does not reach unity and $g^{(2)}(0)$ remains at a value closer to 2 .

\section{CARRIER POPULATION CLAMPING}

Evidence for lasing also arises in the carrier occupation distributions. Figure $6 \mathrm{~b}$ and $6 \mathrm{~d}$ shows population clamping and hole burning for both $\beta=0.01$ and $\beta=1$, when the lasing threshold is reached, regardless of whether the input-output curve exhibits a jump. The data points in Figure $6 \mathrm{a}$ and $6 \mathrm{c}$ indicate the injection currents for the carrier occupation distributions shown in Figure $6 \mathrm{~b}$ and $6 \mathrm{~d}$. In the figures, the gray dotted curves correspond to Fermi-Dirac distributions for similar values of the total hole number and energy. The black curves indicate a quasi-equilibrium condition for the hole populations in the QW. However, clear differences between blue and grey curves indicate non-equilibrium behavior for the QD occupation after the onset of lasing.

\section{EMISSION FROM FEW-QD SYSTEMS}

During the transition to lasing, both Figures $3 \mathrm{~b}$ and $4 \mathrm{~b}$ reveal an interesting feature of $g^{(2)}(0)$ - it drops slightly below unity, which has also been observed experimentally. ${ }^{11}$ The results indicate nonclassical photon statistics within a narrow range of injection currents. Evidence of non-classical light is particularly interesting for applications in quantum information processing. ${ }^{44}$ Emitters based on few QDs are also important in the miniaturization of integrated photonic systems. ${ }^{45}$ Figure 7 displays the behaviors as the QD number is reduced. The effect of a reduced active medium gain is depicted in Figure $7 \mathrm{a}$, where one notes a decrease in laser output at high current (blue and red curves), until lasing is no longer possible (green curve). Figure $7 \mathrm{~b}$ shows that the dip of $g^{(2)}(0)<1$ increases with decreasing QD number. For five QDs in the active region, the $g^{(2)}(0)<1$ region extends indefinitely to high injection currents. For this 5-QD situation, Figure $7 \mathrm{c}$ indicates that there is no increase in coherence time with excitation. We note that current semiconductor-based singlephoton-on-demand sources producing radiation with $g^{(2)}(0)<0.5$ can be realized with individual QD emitters in low- $Q$ optical cavities. Here, one realizes partial antibunching with a finite number of emitters in a higher- $Q$ cavity. With the possibility of increasing the lightmatter coupling due to a high Purcell factor and a small mode volume in active nanoplasmonics, this path may lead to bright alternative nonclassical light sources. 

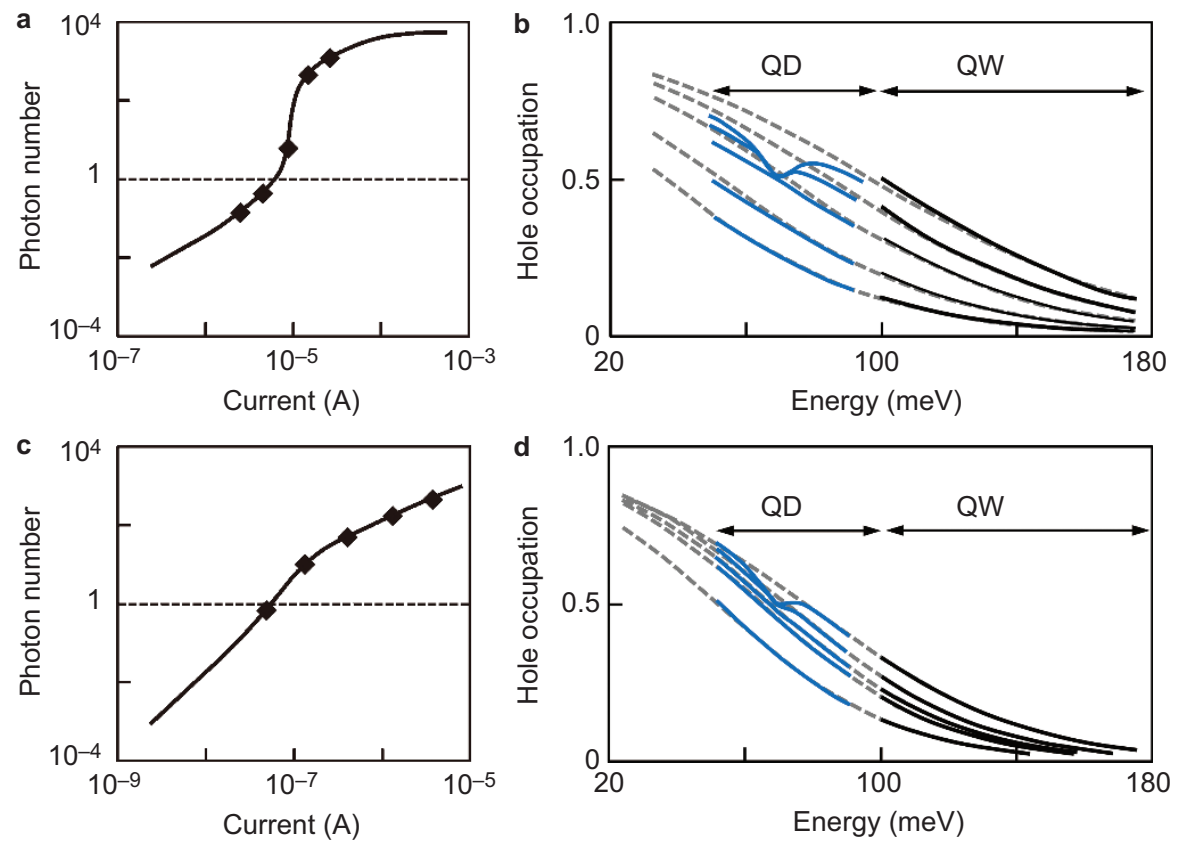

Figure 6 (a) and (c) display input-output curves for 50 QDs with cavity loss $\gamma_{\mathrm{c}}=10^{10} \mathrm{~s}^{-1}$, and inhomogeneous broadening $\Delta_{\text {inh }}=20 \mathrm{meV}$. The points indicate the current for the occupation distributions plotted in (b) and (d). QD, quantum dot; QW, quantum well.

The approximation of light-matter correlations in Equations (3)(5) limits the extent to which the QD number can be reduced within our model because contributions from higher-order light-matter correlations increase with decreasing QD number. We verified the present results by comparison with a model in which correlations are kept to the quadruplet level. To handle the higher-order correlations without substantially increasing computation demands, the comparisons are made without inhomogeneous broadening in the QDs or the effects of the QWs. For the same reason, the effect of super-radiant coupling between the QD emitters is neglected. The comparisons verify the truncation at the doublet level for the parameters considered. We found the super-radiant coupling to be strongly hampered by dephasing and inhomogeneous broadening. However, further investigations are necessary to quantify the increasing role of super-radiant coupling with decreasing QD number.

\section{CONCLUSIONS}

This review is motivated by ongoing discussions involving recent nanolaser experiments, in particular, those related to the criteria for lasing and the determination of a lasing threshold, where much ambiguity exists in cases suggesting thresholdless lasing. Our approach employs a fully quantized laser model to investigate the intensity and coherences of nanolasers. The active medium is treated as inhomogeneously broadened semiconductor QDs embedded in a QW, where carriers are introduced via current injection. Comparisons are made between a typical nanolaser configuration and situations approaching a spontaneous emission factor of unity or few-QD systems. It was found that in general, the combined information of intracavity photon number, coherence time, photon autocorrelation function and carrier spectral hole burning provide a unique and consistent picture for nanolasers in the new regimes of laser operation.

For the situation involving a spontaneous emission factor of unity, the model predicts a smoothing of the intracavity intensity versus pump dependence, which is well known. However, indications remain for the transition from thermal to coherent radiation in the coherence time and in the second-order correlation function, which serve in locating the laser threshold. This finding does not corroborate the notion of thresholdless lasing. While the output intensity benefits from channeling all of the emission into useful optical modes, the emission prior to the onset of stimulated emission will exhibit the
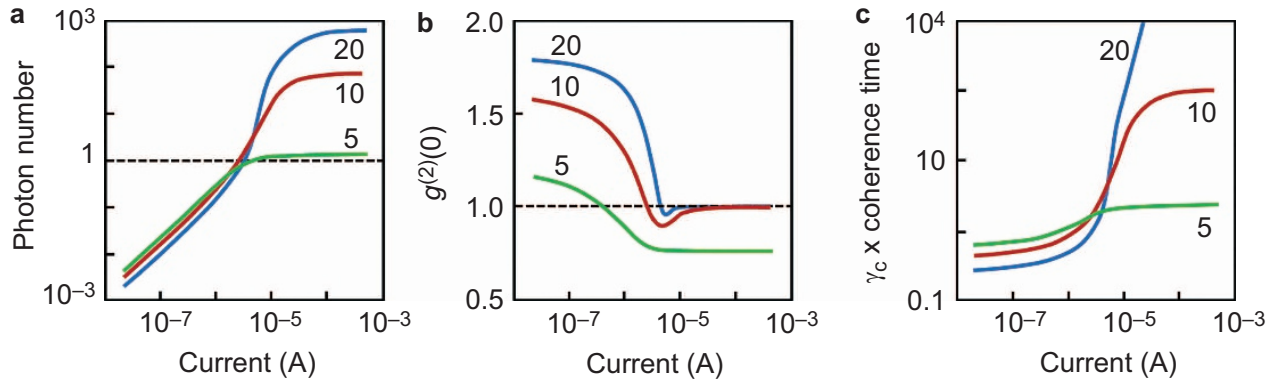

Figure 7 Injection current dependences of (a) photon number, (b) second-order photon correlation and (c) coherence time for a cavity with a spontaneous emission factor $\beta=0.01$ and a photon loss rate $\gamma_{c}=5 \times 10^{10} \mathrm{~s}^{-1}$. The different curves correspond to 20,10 and $5 \mathrm{QDs}$ in the active region. QD, quantum dot. 
intensity fluctuation and low coherence of thermal sources. Still, a $\beta=1$ device is attractive for some applications, such as the next generation of highly efficient lighting, where a spatial concentration of the output beam is desirable, while coherence and intensity fluctuations on the scale governed by the randomness of spontaneous emission are not.

Lastly, preliminary simulations were performed for nanocavities with only a few QDs to provide gain. It is found that as the total number of QDs in the optical cavity is reduced, pronounced evidence for non-classical photon statistics exists for QD numbers as high as ten.

\section{ACKNOWLEDGMENTS}

The present extension of the nanolaser model was developed at the Energy Frontier Research Center for Solid-State Lighting Science, funded by the US Department of Energy, Office of Science, Office of Basic Energy Sciences. The application of this model to the specific cases of unity spontaneous emission and the few-emitter limit was performed under Sandia's LDRD program. FJ and CG acknowledge financial support from the Deutsche Forschungsgemeinschaft. WWC is grateful for the hospitality of the Technical University Berlin and travel support provided by SFB787. FJ acknowledges the hospitality of the Sandia National Laboratories.

1 Yokoyama $\mathrm{H}$, Brorson SD. Rate equation analysis of microcavity lasers. J App/ Phys 1989; 66: 4801-4805.

2 Björk G, Yamamoto Y. Analysis of semiconductor microcavity lasers using rate equations. IEEE J Quant Electron 1991; 27: 2386-2396.

3 Rice PR, Carmichael HJ. Photon statistics of a cavity-QED laser: a comment on the laser-phase-transition analogy. Phys Rev A 1994; 50: 4318-4329.

4 Hinton K, Baliga J, Feng M, Ayre R, Tucker RS. Power consumption and energy efficiency in the internet. IEEE Network 2011; 25: 6-12.

5 Reitzenstein S, Bazhenov A, Gorbunov A, Hofmann C, Münch S et al. Lasing in high-Q quantum-dot micropillar cavities. Appl Phys Lett 2006; 89: 051107.

6 Strauf S, Hennessy K, Rakher MT, Choi YS, Badolato A et al. Self-tuned quantum dot gain in photonic crystal lasers. Phys Rev Lett 2006; 96: 127404.

7 Ulrich SM, Gies C, Ates S, Wiersig J, Reitzenstein S et al. Photon statistics of semiconductor microcavity lasers. Phys Rev Lett 2007; 98: 043906.

8 Xie ZG, Götzinger S, Fang W, Cao H, Solomon GS. Influence of a single quantum dot state on the characteristics of a microdisk laser. Phys Rev Lett 2007; 98: 117401.

9 Reitzenstein S, Böckler C, Bazhenov A, Gorbunov A, Löffler A et al. Single quantum dot controlled lasing effects in high-Q micropillar cavities. Opt Express 2008; 16: 48484857.

10 Nomura M, Kumagai N, Iwamoto S, Ota Y, Arakawa Y. Laser oscillation in a strongly coupled single-quantum-dot-nanocavity system. Nat Phys 2010; 6: 279-283.

11 Wiersig J, Gies C, Jahnke F, Assmann M, Berstermann T et al. Direct observation of correlations between individual photon emission events of a microcavity laser. Nature 2009; 460: 245-249.

12 Fox M. Quantum Optics: An Introduction. Oxford: Oxford University Press; 2006.

13 Gourley PL. Microstructured semiconductor lasers for high-speed information processing. Nature 1994: 371: 571-577.

14 Vahala KJ. Optical microcavities. Nature 2003; 424: 839-846.

15 Lodahl P, van Driel AF, Nikolaev IS, Irman A, Overgaag K et al. Controlling the dynamics of spontaneous emission from quantum dots by photonic crystals. Nature 2004; 430: 654-657.

16 Bergman DJ, Stockman MI. Surface plasmon amplicfication by stimulated emission of radiation: quantum generation of coherent surface plasmon in nanosystems. Phys Rev Lett 2003; 90: 027402.

17 Purcell EM. Spontaneous emission probabilities at radio frequencies. Phys Rev 1946; 69: 681
18 Kleppner D. Inhibited spontaneous emission. Phys Rev Lett 1981; 47: 233-236.

19 Khajavikhan M, Simic A, Katz M, Lee JH, Slutsky B et al. Thresholdless nanoscale coaxial lasers. Nature 2012; 482: 204-207.

20 Yokoyama H. Physics and device applications of optical microcavities. Science 1992; 256: 66-70.

21 De Martini F, Jacobovitz GR. Anomalous spontaneous-stimulated-decay phase transition and zero-threshold laser action in a microscopic cavity. Phys Rev Lett 1988; 60: 1711-1714.

22 Carmichael H, Orozco LA. Quantum optics: single atom lases orderly light. Nature 2003; 425: 246-247.

23 Gies C, Florian M, Gartner P, Jahnke F. The single quantum dot-laser: lasing and strong coupling in the high-excitation regime. Opt Exp 2011; 19: 14370-14388.

24 Grigoriev V, Biancalana F. Resonant self-pulsations in coupled nonlinear microcavities. Phys Rev A 2011; 83: 043816

25 Tame MS, McEnery KR, Özdemir ŞK, Lee J, Maier SA et al. Quantum plasmonics. Nat Phys 2013; 9: 329-340.

26 Ding K, Ning CZ. Metallic subwavelength-cavity semiconductor nanolasers. Light Sci App/2012; 1: e20; doi:10.1038/lsa.2012.20.

27 Pfeiffer M, Lindfors K, Zhang H, Fenk B, Phillipp F et al. Eleven nanometer alignment precision of a plasmonic nanoantenna with a self-assembled GaAs quantum dot. Nano Letter 2014; 14: 197-201.

28 Svelto O. Principles of Lasers. 5th ed. Berlin: Springer; 2010.

29 Blood P. The laser diode: 50 years on. IEEE J Sel Top Quantum Electron 2013; 19: 1503201.

30 DeGiorgio V, Scully MO. Analogy between the laser threshold region and a secondorder phase transition. Phys Rev A 1970; 2: 1170-1177.

31 Elvira D, Hachair X, Verma VB, Braive R, Beaudoin G et al. Higher-order photon correlations in pulsed photonic crystal nanolaser. Phys Rev A 2011; 84: 061802(R).

32 Yamamoto Y, Machida S, Björk G. Microcavity semiconductor laser with enhanced spontaneous emission. Phys Rev A 1991; 44: 657-668.

33 Coldren LA, Corzine SW. Diode Lasers and Photonic Integrated Circuits. New York: Wiley; 1995.

34 Khurgin JB, Sun G. How small can "Nano" be in a "Nanolaser"? Nanoph 2012; 1: 38.

35 Bimberg D, Grundmann M, Ledentsov NN. Quantum Dot Heterostructures. New York: Wiley; 1999.

36 Chow WW, Jahnke F. On the physics of semiconductor quantum dots for applications in lasers and quantum optics. Prog Quantum Electron 2013; 37: 109-184.

37 Gies C, Wiersig J, Lorke M, Jahnke F. Semiconductor model for quantum-dot-based microcavity lasers. Phys Rev A 2007; 75: 013803.

38 Device Simulator. Nextnano: http://www.wsi.tum.de/nextnano (accessed 14 November 2007).

39 Jaynes $\mathrm{E}$, Cummings FW. Comparison of quantum and semiclassical radiation theories with application to the beam maser. Proc IEEE 1963; 51: 89-109.

40 Kira M, Koch SW. Semiconductor Quantum Optics. Cambridge, IL: Cambridge University Press; 2012.

41 Chow WW, Koch SW. Theory of semiconductor quantum-dot laser dynamics. IEEE J Quantum Elect 2005; 41: 495-505

42 Waldmueller I, Chow WW, Young EW, Wanke MC. Nonequilibrium many-body theory of intersubband lasers. IEEE J Quantum Elect 2006; 42: 292-301.

43 Ates S, Gies C, Ulrich SM, Wiersig J, Reitzenstein S et al. Influence of the spontaneous optical emission factor $\beta$ on the first-order coherence of a semiconductor microcavity laser. Phys Rev B 2008; 78: 155319

44 Knill E, Laflamme R, Milburn GJ. A scheme for efficient quantum computation with linear optics. Nature 2001; 409: 46-52.

$45 \mathrm{Wu}$ Q, Turpin JP, Werner DH. Integrated photonic systems based on transformation optics enabled gradient index devices. Light Sci App/ 2012; 1: e38; doi:10.1038/ Isa.2012.38.

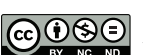

This work is licensed under a Creative Commons Attribution-

NonCommercial-NoDerivs 3.0 Unported License. The images or other third party material in this article are included in the article's Creative Commons license, unless indicated otherwise in the credit line; if the material is not included under the Creative Commons license, users will need to obtain permission from the license holder to reproduce the material. To view a copy of this license, visit http://creativecommons.org/licenses/ by-nc-nd/3.0/ 